Artigo Original

\title{
Liderança no futsal de alta competição: importância dos resultados desportivos
}

\author{
Rui Resende ${ }^{1}$ \\ Rui Gomes ${ }^{2}$ \\ Orlando Vieira $^{3}$ \\ ${ }^{1}$ Centro de Investigação em Desporto, Saúde e Desenvolvimento Humano, Instituto \\ Superior da Maia, São Pedro de Avioso, Portugal \\ ${ }^{2}$ Escola de Psicologia, Centro de Investigação em Psicologia, Universidade do Minho, \\ Braga, Portugal \\ ${ }^{3}$ Instituto Superior da Maia, São Pedro de Avioso, Portugal
}

\begin{abstract}
Resumo: Este estudo realizou-se com 177 atletas de futsal portugueses. Procurou-se verificar se atletas com um passado desportivo de sucesso com os treinadores $(n=62,35 \%)$ se diferenciavam dos atletas sem esse passado desportivo ( $n=115,65 \%$ ), na avaliação da liderança, satisfação e compatibilidade com treinadores. O protocolo de avaliação incluiu medidas de estilos de liderança, satisfação com liderança e compatibilidade treinador-atleta. Os resultados comparativos demonstraram que os atletas com passado desportivo de sucesso avaliaram mais positivamente os treinadores em seis dimensões de liderança, assumiram maior satisfação com o tratamento pessoal e demonstraram maior compatibilidade com os seus treinadores. As análises de regressão demonstram a importância das variáveis de liderança na predição da satisfação com a liderança e da compatibilidade treinador-atleta, com particular importância para a instrução técnica e para o respeito pessoal. Resumindo, comprovou-se a importância dos resultados desportivos na forma como os atletas percebem os respectivos treinadores.
\end{abstract}

Palavras-chave: Liderança. Satisfação Pessoal. Rendimento Atlético.

\section{Leadership in the first division of futsal: the sport results relevance}

Abstract: This study was done with 177 Portuguese athletes of futsal. It was analysed if athletes with a sport success background with their coaches $(n=62,35 \%)$ differed in the evaluation of leadership styles and in the experience of satisfaction and compatibility, from those without that sport success background $(n=115$, $65 \%)$. The evaluation protocol included measures of leadership styles, satisfaction with leadership, and coach-athlete compatibility. Comparative analysis showed that athletes with a sport success background with their coaches' evaluated them more positively in six leadership dimensions, assumed more satisfaction with leadership and showed more compatibility with their coaches. Regression analysis revealed that the leadership variables explained very significant percentages of variance of satisfaction with leadership and coach-athlete compatibility, being particular predictive the technical instruction and the personal respect. This study demonstrates the importance of sport success background in the way athletes evaluate their coaches.

Keywords: Leadership. Personal Satisfaction. Athletic Performance.

\section{Introdução}

O treinador representa uma das figuras com maior influência nas experiências desportivas dos atletas, seja ao nível do impacto que exerce na forma como estes vivenciam a sua atividade, seja ao nível dos resultados que obtêm (HORN, 2008). Neste último caso, uma das formas mais habituais de avaliarmos a eficácia e a capacidade dos treinadores (particularmente os de alta competição) é através do seu sucesso desportivo, nomeadamente em termos dos resultados desportivos alcançados, da classificação obtida numa prova ou num campeonato, dos lugares de pódio, entre outros.
Apesar da relevância desses indicadores no contexto desportivo, alguns autores afirmam que podem comprometer a apreciação do trabalho dos treinadores. Como afirmam Mallett e Côté (2006), o pressuposto de que o treinador é a figura responsável pelo sucesso e insucesso das equipas e dos atletas é no mínimo questionável, existindo evidência prática suficiente que demonstra o oposto. Neste caso, e tomando como exemplo a alta competição, existem casos de treinadores que apesar de não terem alcançado os objetivos desportivos previstos mantiveram um estatuto notável junto dos atletas, assim como existem outros treinadores que 
apesar dos êxitos desportivos não obtiveram grande apreço e respeito por parte dos atletas.

Assim sendo, usar medidas relacionadas com os resultados desportivos para avaliar a eficácia dos treinadores pode ser algo problemático, pois estas podem ser "contaminadas" por um conjunto significativo de fatores não controlados pelos treinadores, como seja, o acaso, a capacidade dos oponentes, a tomada de decisão dos atletas em cada momento da competição, as decisões da arbitragem, entre outros (COURNEYA; CHELLADURAI, 1991). A solução para ultrapassar esse problema poderá passar por utilizar outros indicadores alternativos para avaliar a eficácia dos treinadores. Nesse caso, Chelladurai (2007) sugere a avaliação da percepção de rendimento dos atletas, traduzida pela experiência de satisfação com 0 desempenho individual e coletiva como formas mais adequadas para avaliar o trabalho dos treinadores. Ou seja, mais do que o impacto produzido pelo treinador nos resultados desportivos, importa considerar o seu impacto na experiência desportiva dos atletas e respectivas equipes.

Curiosamente, esta questão também tem sido alvo de debate nos contextos organizacionais mais alargados (como, por exemplo, ao nível das empresas). Na realidade, também aqui existem autores que argumentam que a influência dos líderes no rendimento é mínima e negligenciável, quando comparada com a influência das mudanças sociais, organizacionais e mesmo da própria história (LIEBERSON; O'CONNOR, 1972; PFEFFER,1977). Por isso, alguns autores sustentam que atribuir os resultados organizacionais ao trabalho dos líderes é uma "romanização" simplificadora deste tema (MEINDL; EHRLICH; DUKERICH,1985), pois o desempenho alcançado por um dado grupo ou organização está dependente de um conjunto de variáveis complexas que interagem num sistema externo, dinâmico e aberto (MARION; UHL-BIEN, 2001).

No entanto, essas posições assumidas por todos estes autores encontram um problema difícil de resolver: a evidência empírica. Nesse nível, são vários os estudos que demonstram uma relação entre os comportamentos dos líderes e os resultados alcançados pelas organizações. A título meramente ilustrativo, alguns autores apontam valores de variância explicada nos resultados financeiros das empresas devido ao trabalho dos líderes na ordem dos 14\% (JOYCE; NOHRIA; ROBERSON, 2003), 19\% (MCGAHAN; PORTER,1997), podendo mesmo chegar aos $45 \%$ de variância explicada, dependendo das medidas aplicadas (DAY; LORD, 1988; THOMAS, 1988). Tendo por base estes indicadores, Kaiser, Hogan e Craig (2008) chegam mesmo a prever que as organizações que selecionarem e recompensarem os seus líderes com base no rendimento das respectivas equipes tenderão a ter maior sucesso e a resistir melhor à passagem do tempo. Dito em outras palavras, na perspectiva destes autores, a relação entre liderança e sucesso obtido deve ser estudada, de modo a ser possível perceber quais os fatores que explicam os resultados alcançados pelos líderes com maior impacto no rendimento das respectivas equipes ou organizações. Essa ideia é defendida por Collins (2001), ao afirmar a importância de conhecermos as características dos líderes de sucesso, uma vez que assim estaremos mais perto de explicar o impacto da liderança na performance das equipes/organizações.

A organização deste trabalho encontra-se dentro dessa lógica de entendimento das características de treinadores com e sem passado de sucesso no desporto, analisando a forma como os atletas avaliam os treinadores, tendo por base os resultados desportivos alcançados sob sua orientação. Mais concretamente, avaliamos neste estudo as percepções de atletas de futsal da principal divisão competitiva portuguesa acerca dos estilos de liderança dos respectivos treinadores, dividindo a amostra entre praticantes com um passado desportivo de sucesso com os seus treinadores e aqueles que não alcançaram tal estatuto. Além disso, procuramos igualmente verificar se as experiências de satisfação e compatibilidade com os treinadores variavam em função desta divisão dos participantes.

Neste sentido, este estudo procura verificar se determinado estatuto desportivo alcançado com um treinador estaria condicionado ao modo como seus atletas 0 avaliam, atribuindo-lhe características de liderança distintas em função do sucesso desportivo alcançado. Assim, se a perspectiva da menor importância dos treinadores na influência dos resultados desportivos prevalecer, não será esperado um impacto significativo no modo como os atletas avaliam os 
responsáveis técnicos. Em termos práticos, isto significa um grupo de atletas com e sem passado desportivo de sucesso com os seus treinadores não exibiriam diferenças entre si na avaliação das características de liderança dos seus treinadores. Inversamente, se a perspectiva da maior importância dos treinadores for influenciada pelos resultados desportivos, então poderemos supor que ter sucesso desportivo não é uma variável indiferente à relação treinador-atletas, uma vez que os atletas com sucesso desportivo tenderão a atribuir características de liderança mais positivas aos responsáveis técnicos e a evidenciar experiências desportivas significativamente diferentes dos seus colegas que não atingiram níveis de rendimento tão elevados sob a orientação dos seus treinadores.

Para concretizar este objetivo, realizamos a avaliação dos estilos de liderança através da Escala Multidimensional de Liderança no Desporto (EMLD), (GOMES; RESENDE, 2012), que analisa os comportamentos dos treinadores ao nível transformacional (e.g., influência dos treinadores no desenvolvimento pessoal, psicológico e técnico dos atletas), transacional (e.g., utilização de estratégias de reforço e punição pelos treinadores para influenciar 0 comportamento dos atletas) e tomada de decisão (e.g., utilização de estratégias mais ativas ou passivas no modo como os treinadores tomam decisões na relação com os atletas). Dada a escassez de dados acerca do impacto da liderança transformacional em contextos desportivos, a utilização deste instrumento neste estudo permitiu analisar as dimensões transformadoras da ação dos líderes (BASS, 1985). Assim, ao utilizarmos este instrumento procuramos verificar a possibilidade dos atletas com passado desportivo de sucesso com os seus treinadores poderem avaliar mais facetas transformacionais nos seus treinadores, uma vez que estas tendem a estar associadas a efeitos mais positivos nos colaboradores e nas organizações do que a utilização da liderança transacional (BASS; RIGGIO, 2006).

Além das eventuais diferenças na avaliação dos estilos de liderança, incluímos neste estudo duas medidas relacionadas com a satisfação com a liderança e com a compatibilidade entre treinador-atleta. Ou seja, verificamos se a divisão dos atletas em função do sucesso desportivo alcançado com o atual treinador seria estendida a estas duas dimensões, dando maior abrangência à questão deste estudo. A escolha destas áreas de avaliação deveu-se à influência que ambas exercem nas reações dos atletas face aos comportamentos dos treinadores (KENOW; WILLIAMS, 1999), estando também relacionadas com a experiência dos atletas face às respectivas equipes (CHELLADURAI; RIEMER, 1997; RIEMER, 1998; CHELLADURAI, 1993). Se ocorrerem diferenças nestes dois domínios em função do sucesso desportivo alcançado pelos atletas com os respectivos treinadores, então fortalece-se a ideia de que o sucesso obtido pelos treinadores relaciona-se com o modo como os atletas o avaliam como treinador bem como com o modo como reagem aos seus estilos de liderança.

Assim sendo, este estudo procurou alcançar os seguintes objetivos:

a) Analisar as diferenças ao nível da percepção dos estilos de liderança, da satisfação com a liderança e da compatibilidade treinador-atleta, em função dos resultados desportivos alcançados pelos atletas com o atual treinador; e

b) Analisar as variáveis de liderança preditoras da satisfação com a liderança $e$ da compatibilidade treinador-atleta, em função dos resultados desportivos alcançados pelos atletas com o atual treinador.

\section{Métodos}

\section{Caracterização do estudo}

A investigação empírica de natureza quantitativa que levamos a cabo foi realizada num único momento de coleta de dados (e.g., transversal) (MONTERO; LEÓN, 2007) procurando analisar a relação entre uma variável independente (e.g., passado sucesso desportivo obtido pelos atletas com o atual treinador) e três variáveis dependentes (e.g., estilos de liderança, satisfação com liderança e compatibilidade treinador-atleta), observando também as variáveis de liderança preditoras da satisfação com liderança e compatibilidade treinador-atleta.

\section{Participantes}

Fizeram parte deste estudo 177 atletas do sexo masculino da totalidade das equipas que disputaram o campeonato nacional de futsal da $1^{\underline{a}}$ divisão em Portugal, que representa o escalão máximo deste desporto. Os atletas apresentaram uma média de idades de 25,70 anos $(d p=4,80)$ e uma experiência como atletas que variou entre os 3 e os 31 anos $(M=12,00 ; d p=5,50)$. No que diz respeito aos anos de prática com 0 atual 
treinador, constatou-se que a maioria dos atletas, $54,5 \%$ ( $n=90)$ tinha uma experiência menor ou igual a dois anos. Considerando a principal distinção dos atletas neste estudo, estabeleceu-se como critério fundamental para a inclusão dos atletas na categoria "com passado desportivo de sucesso com o atual treinador" ter obtido o primeiro lugar, em pelo menos, uma das principais provas disputadas pelas respectivas equipes (ex: campeonato nacional, taça de Portugal) bem como ter obtido resultados de relevo ao nível internacional (ex: campeão europeu). Assim sendo, nesta categoria foram identificados $35 \%$ dos atletas $(n=62)$.

\section{Instrumentos de coleta de dados}

Os atletas que participaram neste estudo preencheram um protocolo de avaliação com os instrumentos abaixo descritos.

Questionário demográfico: avaliou variáveis pessoais (como a idade e formação escolar), a carreira desportiva dos atletas (anos de prática e número de internacionalizações como júnior ou sênior) e o tipo de percurso desportivo realizado com o atual treinador (anos de trabalho e resultados alcançados).

Escala de Multidimensional de Liderança no Desporto (EMLD), (GOMES; RESENDE, 2012). Este instrumento avalia nove dimensões de estilos de liderança que se distribuem por três domínios gerais de liderança. O primeiro domínio diz respeito à liderança transformacional, avaliando-se cinco dimensões: (a) visão: tendência de o treinador estabelecer um futuro positivo e desafiador para os atletas; (b) inspiração: comportamentos do treinador no sentido de promover o desejo de sucesso e esforço contínuo dos atletas na concretização das tarefas; (c) instrução técnica: métodos de treino e indicações dadas pelo treinador acerca do que os atletas devem fazer ou como podem corrigir e melhorar as suas capacidades desportivas; (d) respeito pessoal: tendência de o treinador tratar os atletas como pessoas, considerando nas suas decisões os sentimentos e necessidades individuais dos atletas; e (e) apoio pessoal: comportamentos do treinador orientados para o bem-estar dos atletas, evidenciando um interesse em estabelecer relações pessoais francas e informais com os atletas. O segundo domínio diz respeito à liderança transacional, avaliando-se duas dimensões: (f) feedback positivo: comportamentos de reforço e reconhecimento do treinador face ao bom rendimento dos atletas; e (g) feedback negativo: comportamentos de punição e castigo do treinador no sentido de gerir ou controlar os comportamentos inadequados dos atletas. O terceiro domínio diz respeito à tomada de decisão, avaliando-se duas dimensões: (h) gestão ativa do poder: ações do treinador no sentido de promover um maior envolvimento dos atletas no processo de tomada de decisão; e (i) gestão passiva do poder: evitar ou adiar a tomada de decisão, e distanciamento do treinador face às suas responsabilidades quando é necessário resolver problemas importantes. Todos os itens foram respondidos numa escala tipo Likert de 5 pontos (1=Nunca; $5=$ Sempre). A pontuação foi obtida através da soma dos itens de cada subescala, dividindo-se o valor final pelo número de itens que compõem cada dimensão. Valores mais elevados em cada subescala significam maior frequência de comportamentos do treinador na faceta em causa.

Questionário de Satisfação em Atletas (QSA). Trata-se de uma versão traduzida e adaptada por Gomes e Paiva (2010) do Athlete Satisfaction Questionnaire (ASQ), desenvolvida por $\underline{\text { Riemer e }}$ Chelladurai (1998). O QSA é constituído por quinze subescalas, sendo os itens respondidos numa escala tipo Likert de 7 pontos (1=Mesmo nada satisfeito; 7=Extremamente satisfeito). 0 instrumento destina-se a avaliar diferentes aspetos da experiência desportiva dos atletas. Para este estudo, foram considerados quatro domínios fundamentais da satisfação dos atletas com a liderança: (a) satisfação com a utilização das capacidades: avalia a percepção dos atletas acerca da forma como o treinador utiliza e/ou maximiza as suas capacidades e talento individual; (b) satisfação com a estratégia: avalia a percepção dos atletas acerca das estratégias e táticas seguidas pelo treinador durante as competições; (c) satisfação com o tratamento pessoal: avalia a satisfação dos atletas relativamente ao tratamento dado pelo treinador e que, de alguma forma, afeta cada um individualmente e a equipa indiretamente; e (d) satisfação com o treino e instrução: avalia a satisfação dos atletas acerca do treino e instrução fornecidos pelo treinador.

Medida de Compatibilidade Treinador-Atleta (MCTA). Trata-se de uma versão desenvolvida por Gomes e Paiva (2010) a partir dos trabalhos de Kenow e Williams (1999) e Williams et al. (2003). O instrumento é constituído por uma 
dimensão, sendo os itens respondidos numa escala tipo Likert de 9 pontos ( $1=\mathrm{Nada}$ compatíveis; 9=Muito compatíveis). A escala avalia a consonância e compatibilidade entre treinador e atletas em termos desportivos e pessoais, ao nível dos objetivos, ideias, maneira de ser e personalidade. O score é calculado através da soma dos valores dos itens, dividindo depois esse valor pelo número total de itens da escala. As pontuações mais altas significam valores mais elevados de compatibilidade entre treinador e atleta.

Convém ainda referir que a utilização dos resultados obtidos neste estudo foi baseada numa análise prévia dos indicadores de alpha de Cronbach das subescalas, para o total dos participantes. Em praticamente todas as dimensões dos instrumentos utilizados foram observados valores muito aceitáveis, acima de 0,70 (NUNNALLY; BERNSTEIN, 1994). As únicas exceções foram o feedback negativo $(\alpha=0,51) \mathrm{e}$ a gestão passiva do poder $(\alpha=0,68)$ da EMLD. Esta última dimensão foi mantida no estudo devido à sua proximidade do valor aceitável de 0,70 , enquanto que o feedback negativo foi retirado das análises que se seguem.

\section{Procedimentos do estudo}

Este estudo insere-se na atividade de dois centros de investigação portugueses (e.g., Centro de Investigação em Desporto, Saúde e Desenvolvimento Humano e Centro de Investigação em Psicologia), preconizando-se a adoção de procedimentos eticamente definidos na investigação com seres humanos, tendo estes sido seguidos ao longo das várias etapas desta investigação. Assim sendo, o estudo de campo iniciou-se com uma abordagem aos responsáveis técnicos de todas as equipas no sentido de obtermos a concordância para a participação dos atletas no estudo. Após a sua anuência, abordamos os atletas no sentido de procedermos a recolha dos dados. Esta recolha foi efetuada diretamente junto dos atletas, de modo a assegurarmos a melhor taxa de retorno possível. No momento da recolha, começamos explicando, verbalmente e por escrito, os objetivos do estudo e a condição de participação voluntária na investigação, assegurando-se 0 anonimato e a confidencialidade dos dados recolhidos. Apenas os atletas que concordaram com estas condições é que preencheram o protocolo de avaliação. A aplicação dos instrumentos foi sempre realizada na ausência do treinador, dada à temática do estudo. Foram distribuídos 180 protocolos de avaliação tendo sido considerados válidos para este estudo 177 (taxa de retorno de 98\%).

Convém salientar que quando a coleta dos dados foi realizada, todos os atletas tinham um tempo mínimo de trabalho com o atual treinador de pelo menos dois meses, de modo a assegurar que todos os atletas tivessem a oportunidade de conhecer os respectivos treinadores. Este aspecto é particularmente importante para os atletas na condição "sem resultados desportivos com o atual treinador" e a trabalhar pelo "primeiro ano com esse mesmo treinador".

\section{Resultados}

O tratamento e análise estatística foram efetuados no programa SPSS (versão 18.0 para Windows).

\section{Diferenças entre os grupos de atletas com e sem resultados desportivos}

Esta análise procurou verificar a existência de diferenças entre os grupos em análise (atletas com e sem passado desportivo de sucesso com o atual treinador) na percepção acerca dos estilos de liderança, da satisfação com a liderança e da compatibilidade treinador-atleta.

Para a realização desta comparação, começamos por efetuar uma análise exploratória aos dados de modo a observar os pressupostos de aplicação dos testes paramétricos. De um modo geral não foram verificados problemas. Nos casos onde os pressupostos não estavam garantidos, testamos as diferenças utilizando os testes paramétricos e não paramétricos correspondentes, seguindo-se assim as indicações de Fife-Schaw (2006). Os resultados observados de ambos os testes foram similares.

Assim sendo, e começando pelos estilos de liderança, foram identificadas diferenças estatisticamente significativas nas dimensões da EMLD (Wilks' $\lambda=.85, F(9,167)=3.32, p<.01, \eta^{2}$ $=0.15$ ). $O$ valor dos testes univariados apontaram diferenças entre os grupos, observando-se pelos valores médios que os atletas com passado desportivo de sucesso com 0 atual treinador reconheceram existir maior visão por parte do treinador, inspiração, respeito pessoal, apoio pessoal, feedback positivo e gestão partilhada do poder (ver Tabela 1). No que se refere às medidas de satisfação, encontramos também 
valores significativos entre os grupos de estudo (Wilks' $\lambda=.94, F(4,171)=2.69, p<.05, \eta^{2}=.06$ ). Os resultados nos testes univariados indicaram que os atletas com passado desportivo de sucesso com 0 atual treinador demonstraram maior satisfação com o tratamento pessoal (ver Tabela 1). Relativamente às diferenças na dimensão de compatibilidade, a análise de $t$-test para amostras independentes evidenciou diferenças significativas entre os grupos. Assim, os atletas com passado desportivo de sucesso com o atual treinador assumiram maior compatibilidade $(M=6.59, D P=1.46)$ do que os seus colegas sem resultados $(M=5.95, D P=1.62) \quad(t=-2.66 ; 133.8$; p<.01).

Tabela 1. Média, desvio padrão e testes univariados na liderança e na satisfação

\begin{tabular}{|c|c|c|c|}
\hline VARIÁVEL & $\begin{array}{l}\frac{\text { Sem resultados }}{\frac{\text { desportivos }}{(n=115)}} \\
\text { Média (Desvio padrão) }\end{array}$ & $\begin{array}{l}\text { Com resultados } \\
\frac{\text { desportivos }}{(n=62)} \\
\text { Média (Desvio padrão) }\end{array}$ & $F$ valor \\
\hline $\begin{array}{l}\text { Liderança } \\
\text { EMLD: Visão } \\
\text { EMLD: Inspiração } \\
\text { EMLD: Instrução técnica } \\
\text { EMLD: Respeito pessoal } \\
\text { EMLD: Apoio pessoal } \\
\text { EMLD: Feedback positivo } \\
\text { EMLD: Gestão partilhada do poder } \\
\text { EMLD: Gestão passiva do poder }\end{array}$ & $\begin{array}{l}3,41(0,75) \\
3,40(0,78) \\
3,32(0,81) \\
3,54(0,67) \\
2,49(0,79) \\
2,91(0,81) \\
2,26(0,85) \\
2,35(0,72)\end{array}$ & $\begin{array}{l}3,69(0,75) \\
3,82(0,69) \\
3,47(0,77) \\
3,86(0,79) \\
2,87(0,76) \\
3,28(0,74) \\
2,78(0,84) \\
2,31(0,70)\end{array}$ & $\begin{array}{c}(1,175) \\
5,39^{*} \\
12,00^{\star *} \\
1,30 \\
7,73^{\star *} \\
9,67^{\star *} \\
8,48^{\star *} \\
15,11^{\star \star *} \\
0,13\end{array}$ \\
\hline $\begin{array}{l}\text { Satisfação } \\
\text { QSA: Satisfação com utilização das capacidades } \\
\text { QSA: Satisfação com a estratégia } \\
\text { QSA: Satisfação com o tratamento pessoal } \\
\text { QSA: Satisfacão com o treino e instrucão }\end{array}$ & $\begin{array}{l}4,57(1,26) \\
4,58(1,27) \\
4,68(1,34) \\
4,65(1,13)\end{array}$ & $\begin{array}{l}4,71(1,05) \\
4,63(1,70) \\
5,14(1,18) \\
4,69(1,19)\end{array}$ & $\begin{array}{c}(1,174) \\
0,56 \\
0,07 \\
5,02^{*} \\
0,43\end{array}$ \\
\hline
\end{tabular}

\section{Predição da satisfação com a liderança}

Nesta parte do trabalho procuramos predizer a compatibilidade treinador-atleta (MCTA) e a satisfação com a liderança (QSA), a partir da percepção de ambos os grupos de atletas acerca dos comportamentos dos treinadores (e.g., fatores da EMLD). No caso da satisfação com a liderança, foi calculada uma dimensão única a partir dos quatro fatores do QSA, uma vez que estes domínios remetem para o tipo de experiência pessoal e desportiva dos atletas relativamente à liderança dos respectivos treinadores. Ainda, para predições em ambas as dimensões (e.g., compatibilidade e satisfação com a liderança), foi controlada a variável "tempo de trabalho com o atual treinador", uma vez que este fator pode produzir uma influência na relação entre treinador e atletas (ver GOMES; PAIVA, 2010).

As análises de regressão foram realizadas separadamente para os dois grupos de atletas deste estudo (com e sem passado desportivo de sucesso com 0 atual treinador), aplicando-se análises de regressão hierárquica (método "enter"). Neste procedimento, foram observados indicadores de multicolinearidade, constatando-se a ausência deste problema nos modelos testados.

Começando pela predição da satisfação com a liderança nos atletas sem passado desportivo de sucesso com o atual treinador, verificou-se que a variável tempo de trabalho com o atual treinador não foi significativa no modelo de regressão. Já no que se refere às dimensões de liderança, o cenário foi bastante diferente, uma vez que comportamentos de instrução técnica, respeito pessoal e gestão partilhada do poder percebidos pelos atletas, foram preditores significativos da satisfação com a liderança dos treinadores. Neste caso, o modelo final explicou $68 \%$ da variância, tendo sido retirado da análise um caso de outlier (ver Tabela 2).

Quanto à predição da satisfação com a liderança nos atletas com passado desportivo de sucesso com o atual treinador, constatou-se igualmente que a variável tempo de trabalho com o atual treinador não foi significativa no modelo de regressão. No que se refere às dimensões da EMLD, a percepção de instrução técnica (e a visão otimista do futuro, com valores quase significativos) foi a variável preditora da satisfação 
com a liderança, verificando-se que o modelo final explicou 58\% da variância (ver Tabela 2).

Quanto à predição da compatibilidade treinador-atleta no grupo de atletas sem passado desportivo de sucesso com o atual treinador, observou-se uma vez mais que a variável tempo de trabalho com 0 atual treinador não foi significativa no modelo de regressão. No segundo bloco de variáveis, a compatibilidade foi predita pela percepção de instrução técnica, de respeito pessoal e de gestão partilhada do poder. O modelo final explicou $57 \%$ da variância, tendo sido retirados da análise dois casos de outliers (ver Tabela 3).

Tabela 2. Modelos de regressão para a predição da satisfação com a liderança

\begin{tabular}{lcccc}
\hline \multicolumn{5}{c}{ GRUPO DE ATLETAS: SEM RESULTADOS DESPORTIVOS COM O ATUAL TREINADOR } \\
\hline \multirow{2}{*}{ Bloco 1: Anos de trabalho com o atual treinador } & $\boldsymbol{R}^{2}\left(\boldsymbol{R}^{2}\right.$ ajust.) & $\boldsymbol{F}$ & $\boldsymbol{\beta}$ & $\boldsymbol{t}$ valor \\
\hline \multirow{2}{*}{ Bloco 2: Dimensões da liderança } & $0,00(-0,01)$ & $(1,103)$ & $-0,01$ & $-0,12$ \\
\hline EMLD: Instrução técnica & $0,71(0,68)$ & $(10,94)$ & & \\
EMLD: Respeito pessoal & & & 0,48 & $4,93^{* \star \star}$ \\
EMLD: Gestão partilhada do poder & & 0,19 & $2,24^{\star}$ \\
\hline
\end{tabular}

GRUPO DE ATLETAS: COM RESULTADOS DESPORTIVOS COM O ATUAL TREINADOR

\begin{tabular}{lcccc}
\hline & $\boldsymbol{R}^{2}\left(\boldsymbol{R}^{2}\right.$ ajust. $)$ & $\boldsymbol{F}$ & $\boldsymbol{\beta}$ & $\boldsymbol{t}$ valor \\
\hline Bloco 1: Anos de trabalho com o atual treinador ${ }^{2}$ & $0,01(-0,01)$ & $\begin{array}{c}(1,57) \\
0,58\end{array}$ & 0,10 & 0,76 \\
\hline Bloco 2: Dimensões da liderança & $0,65(0,58)$ & $(9,49)$ & \\
EMLD: Visão & & $0,00^{* *}$ & 0,35 & $1,69^{+}$ \\
EMLD: Instrução técnica & & 0,38 & $2,15^{*}$ \\
\hline
\end{tabular}

${ }^{\mathrm{a}}$ Tempo de trabalho com o atual treinador: 0-Até um ano completo de trabalho; 1 -Mais de um ano completo de trabalho

${ }^{*} p<0,05 ;{ }^{* * *} p<0,001 ; \quad{ }^{+} p<0,10$

Tabela 3. Modelos de regressão para a predição da compatibilidade treinador-atleta

\begin{tabular}{lcccc}
\hline \multicolumn{4}{c}{ GRUPO DE ATLETAS: SEM RESULTADOS DESPORTIVOS COM O ATUAL TREINADOR } \\
\hline & $\boldsymbol{R}^{2}\left(\boldsymbol{R}^{2}\right.$ ajust.) & $\boldsymbol{F}$ & $\boldsymbol{\beta}$ & $\boldsymbol{t}$ \\
\hline $\begin{array}{c}\text { Bloco 1: Anos de trabalho com o atual } \\
\text { treinador }\end{array}$ & $0,00(-0,01)$ & $\begin{array}{c}(1,102) \\
0,45\end{array}$ & $-0,07$ & $-0,67$ \\
\hline Bloco 2: Dimensões da liderança & $0,62(0,57)$ & $(10,93)$ & & \\
\hline EMLD: Instrução técnica & & & 0,50 & $4,32^{\star \star *}$ \\
EMLD: Respeito pessoal & & & 0,28 & $2,94^{\star *}$ \\
EMLD: Gestão partilhada do poder & & 0,26 & $2,73^{\star *}$
\end{tabular}

\begin{tabular}{lcccc}
\hline \multicolumn{5}{c}{ GRUPO DE ATLETAS: COM RESULTADOS DESPORTIVOS COM O ATUAL TREINADOR } \\
\hline & $\boldsymbol{R}^{2}\left(\boldsymbol{R}^{2}\right.$ ajust. $)$ & $\boldsymbol{F}$ & $\boldsymbol{\beta}$ & $\boldsymbol{t}$ \\
\hline $\begin{array}{l}\text { Bloco 1: Anos de trabalho com o atual } \\
\text { treinador }\end{array}$ & $0,08(0,06)$ & $\begin{array}{c}(1,56) \\
4,52^{*}\end{array}$ & 0,27 & $2,13^{*}$ \\
\hline Bloco 2: Dimensões da liderança & $0,78(0,73)$ & $\begin{array}{c}(9,48) \\
18,32^{\star * *}\end{array}$ & & \\
EMLD: Inspiração & & & 0,34 & $2,13^{*}$ \\
EMLD: Instrução técnica & & 0,28 & $1,98^{+}$ \\
EMLD: Respeito pessoal & & & 0,25 & $1,86^{+}$ \\
\hline
\end{tabular}

${ }^{\mathrm{a}}$ Tempo de trabalho com o atual treinador: 0-Até um ano completo de trabalho; 1-Mais de um ano completo de trabalho

${ }^{*} p<0,05 ; \quad{ }^{* * *} p<0,001 ; \quad{ }^{+} p<0,10$

Finalmente, na predição da compatibilidade treinador-atleta no grupo de atletas com passado desportivo de sucesso com o atual treinador, foi então possível constatar o valor preditivo do tempo de trabalho com o atual treinador. Atletas 
na condição de "mais de um ano completo de trabalho" com o atual treinador mostraram maior compatibilidade com os respectivos técnicos. Em termos das facetas da EMLD, a inspiração (e dimensões instrução técnica e respeito pessoal, com valores quase significativos) foi a variável preditora da compatibilidade com os treinadores. O modelo final explicou $73 \%$ da variância (ver Tabela 3).

\section{Discussão}

De um modo geral, este estudo demonstra que os treinadores com e sem resultados desportivos de relevância foram avaliados de forma distinta pelos seus atletas, dando assim indicações positivas para a importância desta variável no modo como pode ser analisada a eficácia de treinadores de futsal de alta competição.

Analisando mais em detalhe os resultados, a comparação entre atletas com e sem passado desportivo de sucesso com o atual treinador nas dimensões avaliadas (e.g., EMLD, QSA e MCTA), tornou-se evidente a importância do sucesso desportivo na forma como os atletas avaliaram a liderança dos treinadores, a satisfação que evidenciaram e a própria compatibilidade com os técnicos. Mais concretamente, os atletas com passado desportivo de sucesso com 0 atual treinador avaliaram mais positivamente os estilos de liderança dos seus treinadores, expressando mais ações transformacionais do que o grupo de atletas sem passado desportivo de sucesso (quatro das cinco dimensões neste domínio foram significativas). Neste sentido, torna-se evidente a importância de estudar a liderança transformacional em contextos desportivos, uma vez que esta poderá ajudar a explicar aspetos relacionados com o sucesso desportivo alcançado por treinadores e atletas. De igual modo, estes efeitos positivos também foram generalizados à liderança transacional (com os atletas com passado desportivo de sucesso apontando feedback mais positivo) e à tomada de decisão dos treinadores (com os atletas com passado desportivo de sucesso apontando mais para gestão partilhada do poder). Ou seja, estes resultados demonstram que os efeitos produzidos pelos treinadores com passado desportivo de sucesso nos atletas são bastante positivos e extensos face às dimensões avaliadas pela EMLD. Estes indicadores são significativos uma vez que existem dados da investigação que demonstram que os aumentos nestas facetas de comportamento dos líderes (particularmente das dimensões transformacionais) produzem efeitos muito significativos nos membros dos grupos, nomeadamente ao nível das suas atitudes e rendimento (HARTER; SCHMIDT; HAYES, $2002 \mathrm{e}$ JUDGE; PICCOLO; ILIES, 2004).

Outro dado detectado dos resultados comparativos entre atletas com e sem passado desportivo de sucesso com o atual treinador foi que atletas com sucesso desportivo evidenciaram maior satisfação com 0 tratamento pessoal fornecido pelo treinador e maior compatibilidade com o respectivo treinador. Novamente, a evidência empírica centrada no estudo dos efeitos de líderes com perfis mais positivos de ação (principalmente ao nível da liderança transformacional) demonstrou maiores níveis de comprometimento organizacional (RAI; SINHA, 2000), satisfação e motivação no trabalho (HETLAND; SANDAL, 2003; KOVJANIC; SCHUH; JONAS; QUAQUEBEKE; VAN DICK, 2012) e até mesmo maiores níveis de performance nos membros do grupo (DEGROOT; KICKER; CROSS, 2000; DUMDUM; LOWE; AVOLIO, 2002).

Relativamente às análises de regressão, alguns aspectos parecem comuns em ambos os grupos de atletas. Com efeito, uma das principais dimensões da liderança que emergiu na predição da satisfação com a liderança $e$ da compatibilidade com o treinador, referiu-se à instrução técnica (em três dos quatro modelos testados, alcançou sempre valores significativos). Neste sentido, pode-se afirmar que, independentemente do status desportivo alcançado pelos atletas sob a orientação do treinador, os comportamentos dos treinadores direcionados para o ensino e correção das técnicas da respectiva modalidade, a capacidade para explicarem e treinarem individualmente os atletas nas diferentes competências desportivas representam comportamentos muito significativos na explicação da experiência dos atletas ao nível da satisfação com a liderança $e$ da compatibilidade com 0 treinador. Deve ser salientado o respeito pessoal que, no seu conjunto, foi preditor da satisfação com a liderança e da compatibilidade nos atletas sem resultados desportivos, e alcançou valores quase significativos nos atletas com resultados desportivos. Neste caso, torna-se decisiva a capacidade do treinador assumir comportamentos 
de justiça e equidade na forma como se relaciona com os atletas e a equipe.

Quanto ao que parece diferenciar os dois grupos de atletas, destaca-se sobretudo o valor atribuído pelos atletas sem passado desportivo de sucesso com o atual treinador à gestão partilhada do poder, que foi preditora tanto da satisfação com a liderança, como da compatibilidade. Sob esse ponto de vista, estes atletas conferiram maior peso à possibilidade de serem atendidos nos assuntos importantes relacionados com a atividade desportiva. Já no caso dos atletas com títulos, emergiram duas variáveis preditoras de caráter transformacional: a inspiração (que remete para tendência do treinador estimular 0 alto rendimento nos atletas) e a visão assumida pelo treinador, com valores quase significativos (que sugere uma postura otimista e entusiástica do treinador relativamente ao que os atletas podem alcançar).

$\mathrm{Na}$ relação entre estes dados e a literatura, alguns estudos confirmam a importância de algumas das variáveis preditoras encontradas neste trabalho. Por exemplo, Gomes e Machado (2010) numa investigação com atletas de voleibol, verificaram que as dimensões preditoras da satisfação com a liderança foram essencialmente de natureza transformacional. Num outro estudo levado a cabo por Rowold (2006) com praticante de artes marciais, constatou-se que as dimensões transformacionais dos treinadores (e.g., motivação inspiradora, influência nos ideais, consideração individual) foram variáveis significativas para explicar a eficácia dos treinadores.

Em síntese, este estudo demonstra que a perspectiva de desvalorização da influência dos líderes face aos resultados desportivos poderá não ser muito adequada, principalmente se levarmos em consideração o modo como os atletas avaliam os seus treinadores. Ou seja, os dados obtidos evidenciaram que a percepção dos atletas variou em função do percurso desportivo realizado com os treinadores, fazendo supor a importância dos resultados desportivos na relação estabelecida entre ambas as partes.

Em termos de investigação futura e, dadas as implicações destes resultados, seria interessante realizar estudos com medidas repetidas sobre a percepção dos atletas acerca dos estilos de liderança ao longo da época desportiva, analisando-se assim a eventual flutuação destas avaliações em função do percurso desportivo das equipas. Ao fazê-lo poderemos compreender melhor o real impacto dos líderes nos resultados desportivos e, talvez ainda de forma mais significativa, a influência da obtenção dos resultados desportivos na forma como os atletas percebem e reagem aos estilos de liderança dos seus treinadores.

\section{Referências}

BASS, B. M. Leadership and performance beyond expectations. New York: Free Press, 1985.

BASS, B. M.; RIGGIO, R. E. Transformational leadership (2nd ed.). Mahwah, NJ: Lawrence Erlbaum Associates Inc., 2006.

CHELLADURAI, P. Leadership. In R. N. SINGER; M. MURPHEY; L. K. TENNANT (Eds.), Handbook of research on sport psychology. New York: Macmillan, p. 647-671, 1993.

CHELLADURAI, P. Leadership in sports. In G. TENENBAUM; R. C. EKLUND (Eds.), Handbook of Sport Psychology (3 ed.). Hoboken, New Jersey: John Wiley \& Sons, p. 113-135, 2007.

CHELLADURAI, P.; RIEMER, H. A.A classification of the facets of athlete satisfaction. Journal of Sport Management, Champaign, IL,v. 11, p. 133159, 1997.

COLLINS, J. Good to great. New York: HarperCollins, 2001.

COURNEYA, K. S.; CHELLADURAI, P. A model of performance measures in baseball. Journal of Sport \& Exercise Psychology, Champaign, IL, v. 13, p.16-25, 1991.

DAY, D. V.; LORD, R. G. Executive leadership and organizational performance: Suggestions for a new theory and methodology. Journal of Management, Thousand Oaks, CA,v.14, p. 453464, 1988.

DEGROOT, T.; KICKER, D. S.; CROSS, T. C. A meta-analysis to review organisational outcomes related to charismatic leadership. Canadian Journal of Administrative Sciences, Regina, Saskatchewan,v. 17,n. 4, p. 356-371, 2000.

DUMDUM, U. R.; LOWE, K. B.; AVOLIO, B. J. A meta-analysis of transformational and transactional leadership correlates of effectiveness and satisfaction: An update and extension. IN B. J. AVOLIO; F. J. YAMMARINO (Eds.), Transformational and charismatic 
leadership: The road ahead. Oxford, U.K: JAl/Elsevier, p. 35-66, 2002.

FIFE-SCHAW, C. Levels of measurement. In G. BREAKWELL; S. HAMMOND; C. FIFE-SCHAW; J. SMITH (Eds.), Research methods in psychology. London: Sage, p. 50-63, 2006.

GOMES, A. R.;MACHADO, A. A. Liderança, coesão e satisfação em equipas de voleibol portuguesas: Indicações da investigação e implicações práticas. In M. R. F. BRANDÃO; A. A. MACHADO (Eds.), O Voleibol e a psicologia do esporte. São Paulo: Editora Atheneu, p. 187-218, 2010.

GOMES, A. R.;PAIVA, P. Liderança, compatibilidade treinador-atleta e satisfação no andebol: Percepção de atletas novatos e experientes. PsicoUSF, Itatiba SP, v. 15,n. 2, p. 235-248, 2010.

GOMES, A. R.; RESENDE, R. Assessing leadership styles of coaches and testing the augmentation effect in sport. Submetido para publicação, 2012.

HARTER, J. K.; SCHMIDT, F. L.; HAYES, T. L. Business-unit-level relationship between employee satisfaction, employee engagement, and business outcomes: A meta-analysis. Journal of Applied Psychology, Washington, DC,v. 87, p. 268-279, 2002.

HETLAND, H.; SANDAL, G. Transformational leadership in Norway: Outcomes and personality correlates. European Journal of Work and Organizational Psychology, London, v. 12,n. 2, p. 147-170, 2003.

HORN, T. S. Coaching effectiveness in the sport domain. In T. S. HORN (Ed.), Advances in sport psychology $\left(3^{\text {rd }}\right.$ ed.). Champaign, IL: Human Kinetics, p. 239-267, 2008.

JOYCE, W. F.; NOHRIA, N.; ROBERSON, B. What really works. New York: Harper Business, 2003.

JUDGE, T. A.; PICCOLO, R. F.; ILIES, R. The forgotten ones? The validity of consideration and initiating structure in leadership research. Journal of Applied Psychology, Washington, DC,v. 89, p. 36-51, 2004.

KAISER, R. B.; HOGAN, R.; CRAIG, S. B. Leadership and the fate of organizations.

American Psychologist, Washington, DC,v. 63, p. 96-110, 2008.

KENOW, L.; WILLIAMS, J. Coach-Athlete Compatibility and Athlete's Perception of Coaching
Behaviors. Journal of Sport Behavior, Mobile, AL,v. 22, n. 2, p. 251-259, 1999.

KOVJANIC, S.; SCHUH, S. C.; JONAS, K.; QUAQUEBEKE, N. V.; VAN DICK, R. How do transformational leaders foster positive employee outcomes? A self-determination-based analysis of employees' needs as mediating links. Journal of Organizational Behavior. Chichester, West Sussex,doi: 10.1002/job, 2012.

LIEBERSON, S. A.; O'CONNOR, J. F. Leadership and organizational performance: A study of large corporations. American Sociological Review, London, v. 37, p. 117-130, 1972

MALLETT, C.; CÔTÉ, J. Beyond winning and losing: Guidelines for evaluating high performance coaches. The Sport Psychologist, Champaign, IL, v. 20, p. 213-221, 2006.

MARION, R.; UHL-BIEN, M. Leadership in complex organizations. The Leadership Quarterly, Maryland Heights, v.12, p. 389-418, 2001.

MCGAHAN, A. M.; PORTER, M. E. How much does industry matter, really? . Strategic Management Journal,New York,v. 18, p. 15-30, 1997.

MEINDL, J. R.; EHRLICH, S. B.; DUKERICH, J. $M$. The romance of leadership. Administrative Science Quarterly, New York, v. 30, p. 78-102, 1995.

MONTERO, I.; LEÓN, O.G. A guide for naming research studies in Psychology. International Journal of Clinical and Health Psychology, Granada,v. 7, n. 3, p. 847-862, 2007.

NUNNALLY, J. C.; BERNSTEIN, I. H.

Psychometric theory (3 ed.). New York: McGraw Hill, 1994.

PFEFFER, J. The ambiguity of leadership. Academy of Management Review, New York, v. 2, p. 104-112, 1977.

RAI, S.; SINHA, A. K. Transformational leadership, organizational commitment, and facilitating climate. Psychological Studies, New York,v. 45, n. 1-2, p. 33-42, 2000.

RIEMER, H.; CHELLADURAI, P. Development of the athlete satisfaction questionnaire (ASQ).

Journal of Sport \& Exercise

Psychology,Champaign, IL, v. 20, p. 127-156, 1998.

ROWOLD, J. Transformational and transactional leadership in martial arts. Journal of Applied 
Sport Psychology, Washington, DC,v. 18, p. 312325, 2006.

THOMAS, A. Does leadership make a difference to organizational performance? Administrative Science Quarterly, New York, v. 33, p. 388-400, 1988.

WILLIAMS, J.; JEROME, G.; KENOW, L.;

ROGERS, T.; SARTAIN, T.; DARLAND, G. Factor structure of the coaching behavior questionnaire and its relationships to athlete variables. The Sport Psychologist, Champaign, IL, v. 17, p. 1634, 2003.

\section{Endereço:}

Escola de Psicologia, Campus de Gualtar

Rui Gomes

4710-057-Braga

Portugal

Telefone: +253.604 .232$

Fax: +253.604.224

e-mail: rgomes@psi.uminho.pt

Recebido em: 25 de abril de 2012.

Aceito em: 15 de maio de 2013.

\section{(c) (†)}

Motriz. Revista de Educação Física. UNESP, Rio Claro, SP, Brasil - elSSN: 1980-6574 - está licenciada sob Creative Commons - Atribuição 3.0 